\title{
How does a chain-extended polylactide behave?: a comprehensive analysis of the material, structural and mechanical properties
}

\author{
A. Jaszkiewicz • A. K. Bledzki • R. van der Meer • \\ P. Franciszczak $\cdot$ A. Meljon
}

Received: 6 May 2013/Revised: 11 September 2013/Accepted: 8 April 2014/

Published online: 22 April 2014

(C) The Author(s) 2014. This article is published with open access at Springerlink.com

\begin{abstract}
The presented contribution deals with the material properties of chainextended polylactide. In the course of this study, two different functional additives were used, namely an epoxidized and a maleated styrene-acrylic copolymer. Both additives were compounded together with polylactide using a conventional twinscrew extruder, and were then injection moulded to standardized testing specimens. The main focus of the investigation is on structural changes as well as the mechanical performance, e.g. crack propagation and arresting mechanisms that are affected by reactive chain extension. The first section of the experimental part consists of results regarding modifications achieved on the macromolecular level. Based on size exclusion chromatography and DSC-OIT experiments, different structural changes and their influence on the material behaviour are presented. Subsequently, a comprehensive analysis of the quasi-static tests and the impact strength of notched and unnotched specimens was performed, and correlated with the following fatigue experiments. A discussion concerning morphological aspects and finally a correlation to the fracture surface topography after fatigue test completes the experimental part.
\end{abstract}

\footnotetext{
A. Jaszkiewicz $\cdot$ A. K. Bledzki

Institut für Werkstofftechnik, Universität Kassel, Mönchebergstr. 3, 34125 Kassel, Germany
}

Present Address:

A. Jaszkiewicz

BASF SE, Carl-Bosch-Str. 38, 67056 Ludwigshafen, Germany

\author{
A. K. Bledzki · P. Franciszczak · A. Meljon ( () \\ Faculty of Mechanical Engineering and Mechatronics, Institute of Materials Science, West \\ Pomeranian University of Technology Szczecin, Piastów 19 Avenue, 70-310 Szczecin, Poland \\ e-mail: ameljon@zut.edu.pl \\ R. van der Meer \\ BASF Nederland B.V, Innovatielaan 1, 8466 SN Nijehaske, The Netherlands
}


Keywords Polylactide $\cdot$ Chain extension - Structural modification · Crack arresting - Mechanical performance

\section{Introduction}

One of the process-relevant concerns regarding polylactide is its tendency to result in polymer degradation during processing and its extremely low melt strength. Literature also underlines that this effect results in undesirable phenomena (e.g. a deterioration of the processability and mechanical performance), often limiting the potential of new applications [1, 2]. In addition, commercial polylactides display characteristic brittle fracture behaviour in the range of typical service temperatures. This material-specific phenomenon is principally due to the aliphatic and substantially amorphous structure of the polymer. Besides this, most bio-based polyesters are characterized by acceptable strengths, but simultaneously extremely high stiffness. The opposing strength and stiffness, in turn, determine the deformation performance. This unfavourable relationship leads to supplementary embrittlement, which further reduces the material toughness. Such material behaviour is distinguished by increased cracking, resulting in premature catastrophic failure. Currently, an intensive research study is being performed which examines to what extent these disadvantages can be omitted by the use of additives $[1,2]$, fillers [3-5] or reinforcing fibres [6-8]. A comprehensive statement about the cracking and fracture behaviour under dynamic (impact), or cyclic-dynamic loading (fatigue) has not yet been made.

In this contribution, injection moulding type of polylactide is the subject of the investigations in terms of its material properties when combined with an epoxidized chain extender. The resulting mechanical performance is analysed with regard to the quasi-static properties, the impact toughness and the crack resistance during fatigue. First, the influence of chain extension on the structural properties after compounding PLA with an epoxidized functional additive is shown. Afterwards, the resulting mechanical properties are discussed. In the last section, some general conclusions regarding the morphological changes in the chain-extended polylactides after the fatigue test will be drawn.

\section{Experimental section}

\section{Materials}

Polylactide manufactured by NatureWorks LLC/USA and named PLA 3051D was used for the investigation. It was supplied in the form of semi-crystalline pellets. According to processing guidelines, the polymer should be processed in the temperature range $190-205{ }^{\circ} \mathrm{C}$. Further material parameters are listed in Table 1.

As part of the study, two multifunctional additives were used. CESA-extend is a MB based on an epoxidized styrene-acrylic copolymer chain extender, of which the 
Table 1 Basic properties of PLA 3051D

\begin{tabular}{lll}
\hline Property & Unit & PLA 3051D \\
Melt temperature & ${ }^{\circ} \mathrm{C}$ & $150-165$ \\
MFR (210/216) & $\mathrm{g} / 10 \mathrm{~min}$ & 13 \\
Density & $\mathrm{g} / \mathrm{cm}^{3}$ & 1.25 \\
D-Lactide content & $\%$ & $3.7-4.6$ \\
Residual monomer & $\%$ & 0.30 (maximum) \\
\hline
\end{tabular}

epoxy functionality equals 9 . The chain extender was provided in the form of a solid masterbatch composed of a carrier polymer (commercial polylactide) and Joncryl ADR4368S (produced by BASF SE) which acts as a functional constituent. Joncryl ADR4368S is characterized by a weight average molecular mass of $6,800 \mathrm{~g} / \mathrm{mol}$, a glass transition temperature of $54{ }^{\circ} \mathrm{C}$ and an epoxy weight equivalent equalling $280 \mathrm{~g} / \mathrm{eq}$. The masterbatch was delivered by Clariant Masterbatches. In this paper, the abbreviation "CESA" will be used.

Joncryl ADR3229 is an additive functionalized with maleic acid anhydride (MAH). The main structure of the additive is similar to that of Joncryl ADR4368S (styrene copolymer). The MAH-functionality is 6, the weight molecular mass equals $8,300 \mathrm{~g} / \mathrm{mol}$ and the MAH weight equivalent amounts to $450 \mathrm{~g} / \mathrm{eq}$. Joncryl ADR3229 has a higher glass transition temperature which equals approx. $110^{\circ} \mathrm{C}$. Throughout this thesis, the generalized name "Joncryl" will be employed $[9,10]$.

\section{Processing conditions}

The chain extender was added to the polymer during compounding. For this purpose, the co-rotating twin-screw extruder Haake PTW Polylab 25/32p with a screw diameter of $25 \mathrm{~mm}$ and a nominal screw length of $L=32 \mathrm{D}$ was used. Prior to this step, PLA 3051D was dried at $110{ }^{\circ} \mathrm{C}$ overnight in a convection oven (max. moisture content $150 \pm 50 \mathrm{ppm}$ ). Then, the pellets were manually mixed with a chain extender and inserted into the extruder in form of a dry-blend through a sidefeeder made by Gebrüder Haake GmbH (type MF 1). To minimize the water uptake from the atmosphere, the granules were added directly after drying into a feed hopper and the cover was immediately closed. The throughput was set at a constant screw velocity of $100 \mathrm{rpm}$ and a constant torque of $80 \mathrm{Nm}$ to keep the shear forces comparable by trend. Exceptions are experiments done with varying screw velocities that were performed on unmodified PLA. Here, the screw speed was set in the range 50-400 rpm. The nominal temperature upon entry was set at $200{ }^{\circ} \mathrm{C}$, and was kept at $210{ }^{\circ} \mathrm{C}$ in the following seven zones. The pellets produced in this way were used for injection moulding and for further analysis concerning the structural and mechanical properties.

The injection moulding machine Klöckner Ferromatik FM 85, manufactured by Klöckner Ferromatik, was used to produce test specimens. The screw has a screw diameter of $40 \mathrm{~mm}$ and a clamping force of $850 \mathrm{kN}$. The L/D ratio is 21 . The applied temperature profile can be found in Table 2 . 
Table 2 Temperature profile during injection moulding

\begin{tabular}{lcr}
\hline Zone & Unit & Value \\
\hline Zone 1 (entry) & ${ }^{\circ} \mathrm{C}$ & 50 \\
Zone 2 & ${ }^{\circ} \mathrm{C}$ & 180 \\
Zone 3 & ${ }^{\circ} \mathrm{C}$ & 190 \\
Zone 4 & ${ }^{\circ} \mathrm{C}$ & 200 \\
Zone 5 (nozzle) & ${ }^{\circ} \mathrm{C}$ & 190 \\
Mould temperature & ${ }^{\circ} \mathrm{C}$ & 40 \\
\hline
\end{tabular}

The injection pressure was set in accordance with the cited ISO standard. The resulting, constant cavity pressure for all trials was 450 bar. The constant injection rate of $200 \mathrm{~mm} / \mathrm{s}$ in the critical cross-section of the sample was used. All materials were dried at $110{ }^{\circ} \mathrm{C}$ overnight prior to injection moulding.

Evaluation of structural and mechanical properties

The analysis of the molecular weight was completed using size exclusion chromatography. Accordingly, the polylactide was dissolved in dichloromethane (total volume was $100 \mu \mathrm{L}$ ). To ensure a constant flow of the mobile phase, an isocratic pump, type Agilent 1100, was used. A Wyatt Optilab 903 interferometric refractometer and a MALLS Dawn F laser photometer (both Wyatt Technology) were employed as detectors in the series. For the estimation of molecular masses, two PLGel 5 microns MIXD C-columns were used. The separation took place at room temperature. The calibration of the MALLS detector was calculated using the toluene reference with the normalization of polystyrene $(30,000 \mathrm{~g} / \mathrm{mol})$.

The calorimetric determination was performed using the differential scanning calorimeter Q1000 made by TA Instruments. Due to the fact that the thermooxidative stability varied with increasing molecular weight, dynamic oxidative induction temperature (OIT) measurements were performed. The specimen, which weighed about $10 \mathrm{mg}$, was first heated under a nitrogen atmosphere at $10 \mathrm{~K} / \mathrm{min}-$ $200{ }^{\circ} \mathrm{C}$. After $5 \mathrm{~min}$ of isothermal holding, the sample was cooled down to $0{ }^{\circ} \mathrm{C}$. Thereby, the artefacts of the thermal history were eliminated. In the third cycle, the material was heated in an oxygen atmosphere at $10 \mathrm{~K} / \mathrm{min}-350{ }^{\circ} \mathrm{C}$. The samples were analysed in unsealed aluminium pans.

The manufactured test specimens were subjected to tensile and flexural testing using a Zwick/Roell UPM 1446 universal testing machine. The test results were summarized with the aid of the computer-supported software testXpert ${ }^{\circledR}$. The following mean values consist of an average of at least six separate measurements. The test parameters are shown in Table 3.

To investigate the toughness of the composites, instrumented Charpy impact tests according to ISO 179-II (with and without A-notch) were carried out. The A-notch was realized with a NOTCHVIS notching machine from CEAST.

The computer-supported, load-controlled, single-axle load increase test (MTS 810 Hydrodynamic Pulser) was used to determine the dynamic mechanical 
Table 3 Testing parameters of the quasi-static tests

\begin{tabular}{llll}
\hline & Unit & Tensile test & 3-point-bending test \\
\hline ISO standard & - & ISO 527-1 & ISO 178 \\
Specimen geometry & - & $1 \mathrm{~A}$ & $80 \times 10 \times 4 \mathrm{~mm}$ \\
Testing velocity & $\mathrm{mm} / \mathrm{min}$ & 5 & 5 \\
Testing velocity E-modulus & $\mathrm{mm} / \mathrm{min}$ & 1 & \\
Measuring range E-modulus & $\%$ & $0.05-0.25$ & \\
\hline
\end{tabular}

properties under cyclic loading conditions. Characteristics such as the fatigue modulus, mechanical damping and number of cycles to failure were determined so as to examine rupture. Samples clamped on both sides (type 1A) were loaded with a sinusoidal stress. An initial loading level (maximum stress) of $15 \mathrm{MPa}$ was used in the tension-tension modus. A test frequency of $5 \mathrm{~Hz}$ was chosen to prevent any warming of the specimen surface. After an oscillation interval of 5,000 cycles, the maximum stress was increased by $5 \mathrm{MPa}$. The $R$-ratio (proportion of minimum to maximum stress during one fatigue cycle) remained constant at $R=0.1$. Throughout the experiment, the force deformation behaviour was recorded simultaneously by the test software.

The test specimens (type 1A in line with ISO 527) for mechanical analyses were manufactured in an injection moulding process in accordance with ISO 294-I, using a normalized mould with a Z-melt distributor. The test samples for flexural and impact tests were mechanically cut from the parallel length of the standardized $1 \mathrm{~A}$ test bar in accordance with ISO 2818. All mechanical tests were performed at ambient temperature after $16 \mathrm{~h}$ conditioning at $23{ }^{\circ} \mathrm{C}$ and $50 \%$ relative humidity. The mean value of at least six repetitions for each trial was calculated.

\section{Results and discussion}

Size exclusion chromatography (SEC) results

The results from the SEC show two different material behaviours. Modifying PLA with the maleated chain extender (Joncryl) results in no visible change of the molecular weight. For instance, the weight average molecular mass of polylactide with $2 \mathrm{wt} \%$ Joncryl does not change compared to the extruded unmodified PLA.

In contrast, adding CESA to polylactide causes a noticeable chain extension. Considering the multi-functionality of CESA (number of functional groups per molecule equals 9), a non-linear chain extension (chain branching) can be assumed. An equivalent concentration of this additive leads to a significant increase of the molecular weight by the factor 3.2. Reference PLA after extrusion displays a molecular weight of $75,000 \mathrm{~g} / \mathrm{mol}$ compared to $240,400 \mathrm{~g} / \mathrm{mol}$ at $2 \mathrm{wt} \%$ CESA content. This is evident when examining the obtained values of the weight average molecular masses (Fig. 1). 


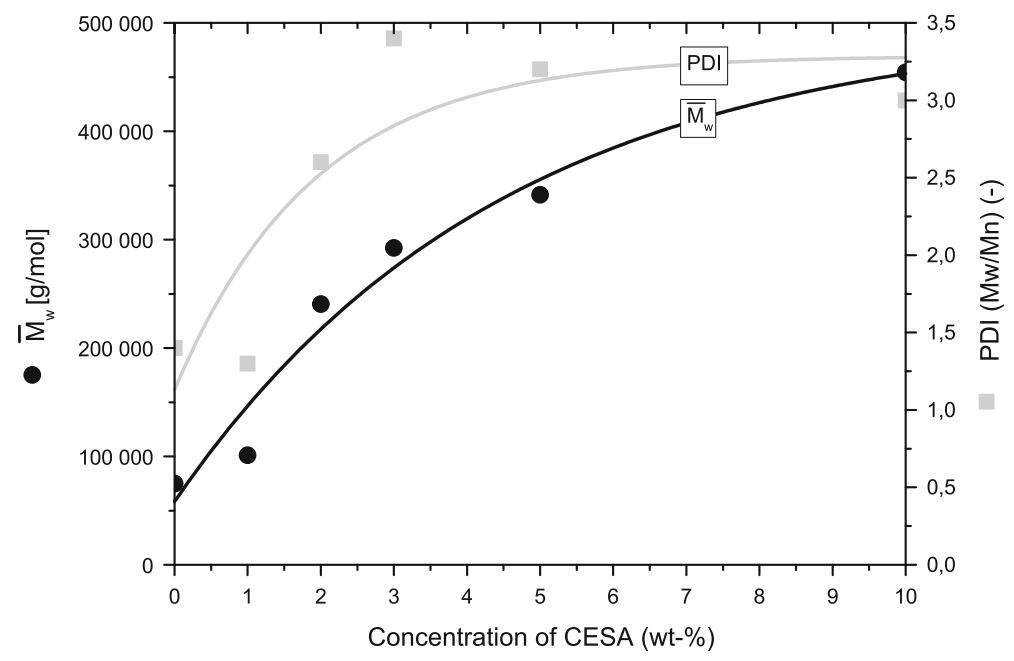

Fig. 1 Weight average molecular mass and the polydispersity of PLA with CESA

The SEC analysis verifies the influence of epoxidized chain extenders on the molecular weight. It was quantitatively confirmed that even very low concentrations of CESA increase the molecular weight drastically. The customized structure of the multifunctional chain extender favours the formation of highly branched chains. Furthermore, a chain extension reaction with maleic anhydride functionalised Joncryl could not be detected, indicating no chemical affinity between these two components.

Crystallization behaviour and thermo-oxidative ageing of chain-extended polylactide

The thermo-oxidative degradation of polymeric structures already takes place during processing, where the melt is exposed to an elevated temperature and oxygen. The effects of thermo-oxidative ageing can therefore be prevented by adding stabilizers that delay degradation, or by means of selective modification of the terminal groups [11]. The OIT, as one of the assessment methods for the thermal stability, can be quantitatively measured by means of a DSC-OIT analysis.

Various screw velocities were used to determine the OIT of PLA 3051D pellets. Figure 2 shows no significant deviations of the OIT measured for virgin PLA. An exemplary thermogram of the DSC-OIT results of pellets without chain extender is shown in Fig. 2.

The resistance against thermo-oxidative degradation remains apparently unaffected by the respective processing parameters, and seems to be comparable for all analysed materials. The relatively small difference between virgin PLA and extruded materials can be assigned to the mined molecular weight (Table 4).

It is, however, noticeable that the crystallinity of the extrudates differs from the completely amorphous reference PLA 3051D. Vegt [12] relates this effect to the 


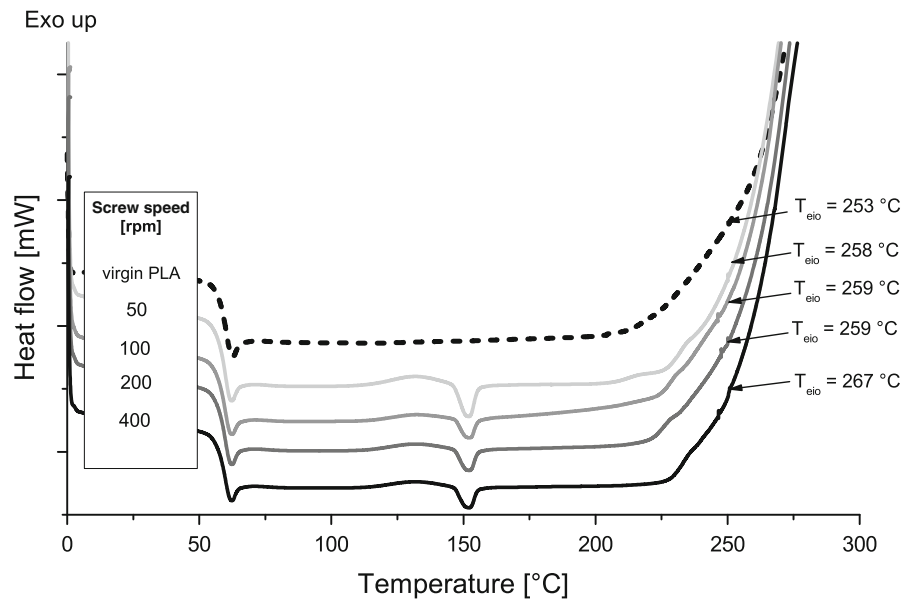

Fig. 2 Evaluation of the dynamic OIT measurements of PLA 3051D (3rd heating cycle) $T_{\text {eio }}-$ extrapolated initial temperature of the oxidation, the intersection of the tangents before and during oxidation

Table 4 SEC analysis of extruded PLA pellets

\begin{tabular}{llll}
\hline Screw velocity $(\mathrm{rpm})$ & $\bar{M}_{\mathrm{w}}(\mathrm{g} / \mathrm{mol})$ & $\bar{M}_{\mathrm{n}}(\mathrm{g} / \mathrm{mol})$ & $\bar{M}_{\mathrm{w}} / \bar{M}_{\mathrm{n}}(-)$ \\
\hline 50 & 82,000 & 58,000 & 1.4 \\
100 & 75,000 & 54,000 & 1.4 \\
200 & 75,000 & 49,000 & 1.5 \\
400 & 71,000 & 49,000 & 1.4 \\
Virgin PLA & 91,000 & 61,000 & 1.5 \\
\hline
\end{tabular}

partially degraded chains. Due to the increasing fraction of cleaved chains, the number of disturbing end groups increases. In the present case, it can be seen as a "nucleating agent", which eventually promotes crystallization. In contrast to virgin PLA, the melting peak of extruded polylactides is well recognizable, in spite of the extinguishing of the thermal history in the first heating cycle. The influence of minor dosages of short-chain polylactides on the crystallization behaviour has been investigated by Siebert-Raths and Endres [13]. Their results confirmed the crystallization behaviour verified within the course of this study. However, counter to the presented work, the authors used highly pure, commercial PL(L)A, which led to the formation of stereocomplexes due to heterogeneous crystallization. The fact that the presence of polymer chains of different lengths stimulates the crystallization of polylactide can be demonstrated analysing the OIT traces of PLA modified with CESA (Fig. 3).

Consequently, a clear correlation between the dosage of CESA and the obvious peaks of secondary crystallization can be seen. Resulting from the broader distribution of the chain lengths, the temperature of secondary crystallization tends 


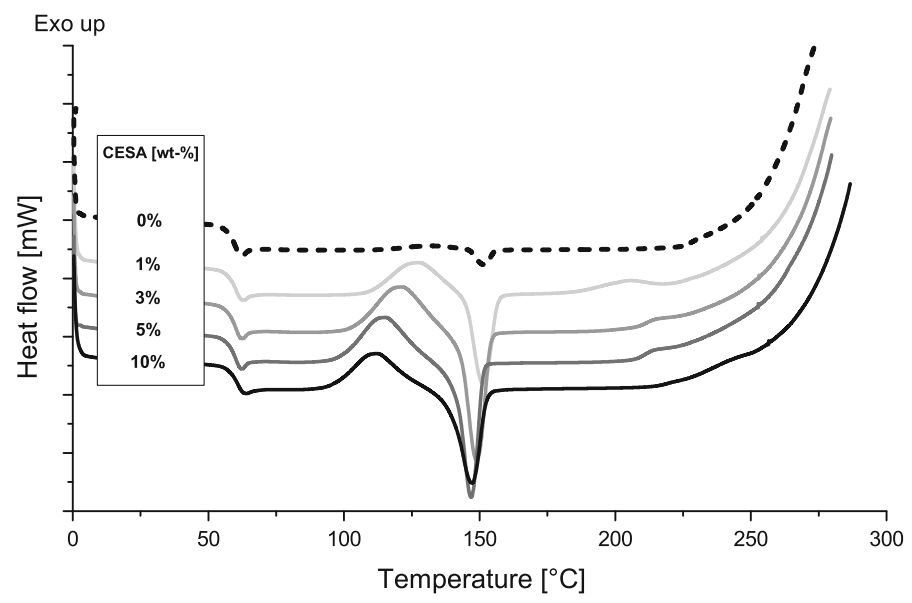

Fig. 3 Evaluation of the dynamic OIT measurements of PLA 3051D with CESA (3rd heating cycle)

to decrease. Also, the melting enthalpy changes with the increasing content of chain extender. The higher melt enthalpy can be directly attributed to the enhanced crystallinity (Fig. 4).

Using chain extender results in increased molecular weight and lower chain mobility, which normally appears in decreased crystallization rate. However this investigation shows clearly that the use of chain extender reduced crystallization temperature and increased crystallinity. This indicates the improvement in crystallization rate, which may be a result of chain extender acting as a nucleating agent, which accelerates the formation of crystal structures significantly. The slight reduction of the melt temperature can also be attributed to imperfect crystal structures. The re-building of the molecular weight has a clear effect on the oxidative induction temperature. The obtained OIT values tend to increase in correlation with a larger CESA content. This dependency is illustrated in Fig. 5.

Quasi-static mechanical properties of chain-extended polylactides

The results from Fig. 6 confirm the suspicions that the basic material characteristics of polylactides modified with MAH-functionalized chain extenders show only a negligible change. The slight decrease in strength can mainly be explained by a progressive separation of the two components. The findings, so far, show no interaction between the anhydride and the PLA terminal groups. In contrast, CESA is a masterbatch based on a PLA carrier, so both components are thermodynamically miscible. This was confirmed by an accompanying DMA investigation (not shown here). In the presented case, CESA leads to a formation of branched macromolecules which have a detectable and beneficial effect on the strength.

In comparison to virgin PLA, the tensile and flexural strengths increase by approx. $15 \%$ when the CESA content equals $10 \mathrm{wt} \%$. It is more likely that the chain extension reduces the number of chain ends, which, in turn, act as stress 


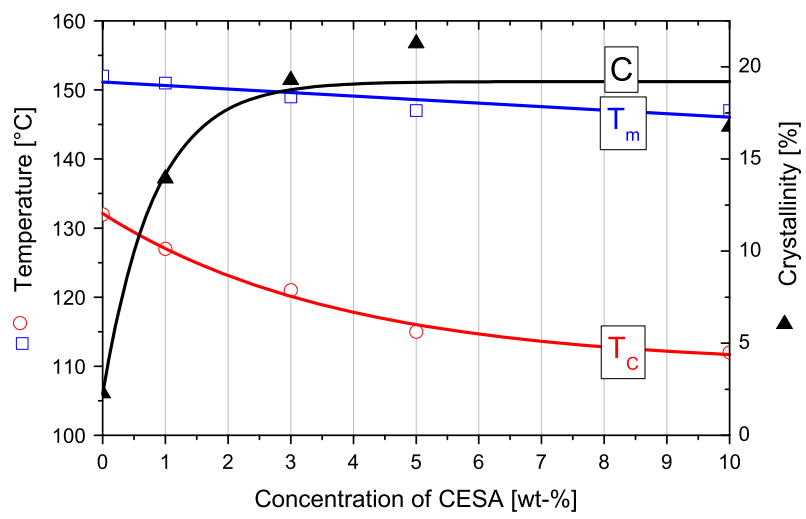

Fig. 4 Evaluation of the transition temperatures and crystallinity of PLA 3051D with CESA (3rd heating cycle) $C$-crystallinity [\%], $C$ was calculated from the ratio between the determined and the theoretical enthalpy of a $100 \%$ crystalline polylactide $\left(\mathrm{H}_{100 \% \mathrm{PLA}}=94 \mathrm{~J} / \mathrm{g}\right.$ [14] $) T_{\mathrm{m}}$-extrapolated melting temperature representing the maximum of the melting peak $T_{\mathrm{C}}$ - extrapolated cold crystallization temperature representing the peak maximum of secondary crystallization

Fig. 5 Correlation of the extrapolated initial temperature of the oxidation of PLA with CESA

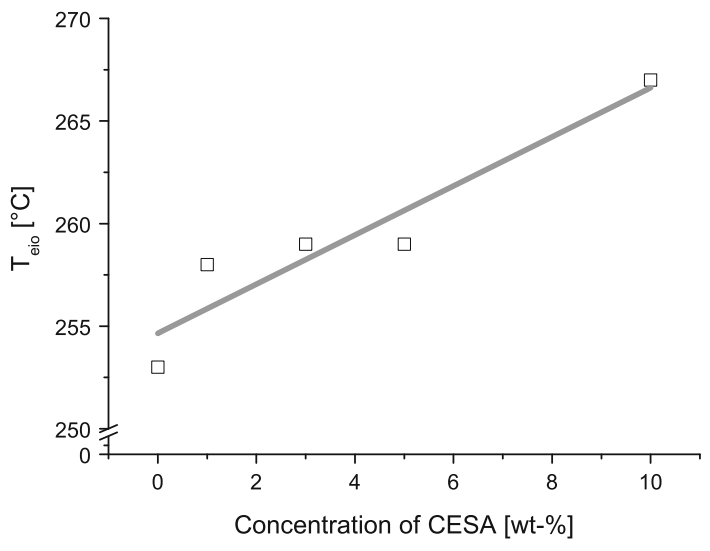

concentration. Moreover, the branches have stronger binding forces and therefore produce higher strengths. Also, the possibility of a higher orientation of the molecules can be excluded when taking the highly branched structures into account.

In sum, it is clear that the new morphological structures act as crack-arresting mechanisms, most efficiently when they are present in the form of large (highly branched), quasi-homogenously distributed accumulations. Similar micromechanical effects have already been discussed by Michler [15]. Also, Vegt [16] describes such a statement as a very plausible explanation. Both authors relate to known petrochemical plastics and mixtures thereof. The presented study could thus prove the basic findings on the basis of new polymers from renewable resources. The deformation behaviour remains almost unaffected. For instance, all modifications with CESA show a strain-at-break of about 3.5-4.5\% in the tensile test. 

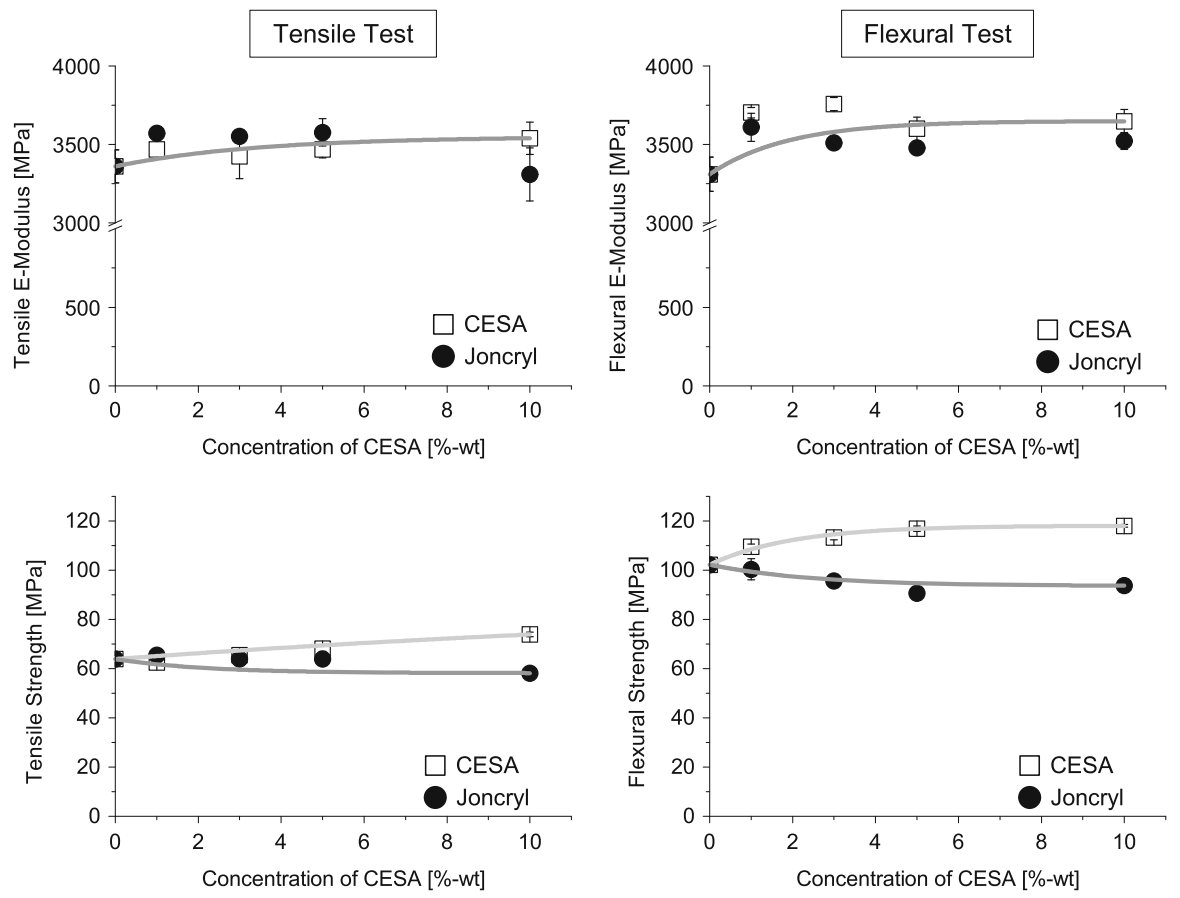

Fig. 6 Results of quasi-static tensile and bending tests

Impact toughness and fatigue behaviour

As was the case for the discussion concerning the fracture mechanisms of modified PLA, the results regarding the impact toughness are of particular importance. If the assumptions regarding the crack-arresting mechanisms in the case of CESA are correct, these effects must be even more evident in the Charpy impact test. In fact, the values of the notched impact strength support the statement that the obtained branches contribute to the increase in the material resistance to impact loads (Fig. 7).

It was assumed that the branched PLA chains form statistically evenly distributed fractions. Otherwise, the accumulation of unevenly distributed branched molecules would lead to a significant spread of the values from the tensile and bending tests. The influence of the epoxidized chain extender on the A-notch impact strength is, however, noticeable at $5 \mathrm{wt} \%$. This means that the crack propagation on the tip can only be arrested when higher concentrations of CESA are on hand. It is also obvious that the increasing content of Joncryl leads to no detectable improvement.

In conclusion, it can be said that the notch impact strength is improved mainly by the formation of long, branched chains. Basically, two independent mechanisms that inhibit the crack formation play a crucial role. First, there is the reduction of stressconcentrated chain ends. This effect is mainly caused by the increase in the molecular weight. In consequence, CESA binds the reactive terminal groups to 


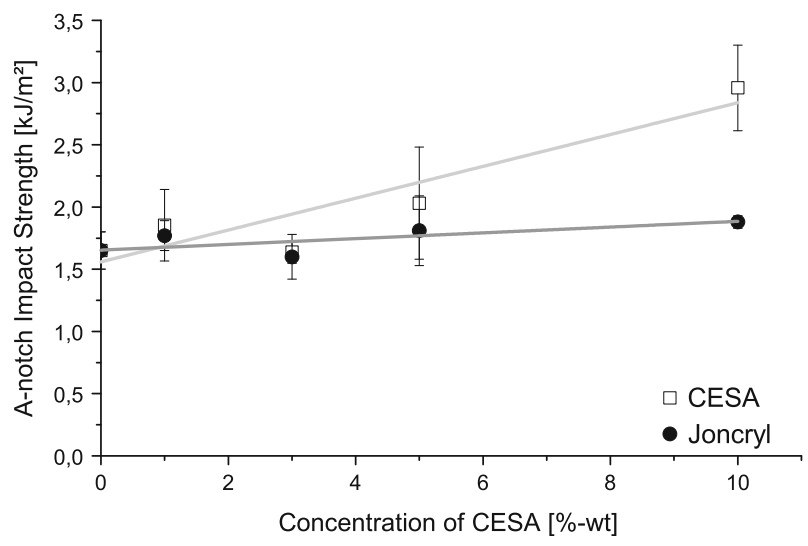

Fig. 7 Results from the Charpy impact test (A-notch, $T=23{ }^{\circ} \mathrm{C}$ )

larger macromolecules, leading to a formation of quasi-homogenously distributed local regions of differing strengths (accumulation of branched chains). The local change of the material toughness results in a rounding of the crack tip, and, thus, leads to an increase of plastic stretching in front of the progressing crack. Another explanation is the transfer of emerging microcracks in the stress-relieving surface configuration [17].

With regard to the influence of crack-arresting mechanisms in brittle polymers, the notched impact strength only finds limited application. One of the concerns is, for instance, the way the notch is produced. In notched specimens, the targeted stress concentration occurs at the notch tip, which defines the starting point of crack propagation in ductile plastics. Amorphous polyesters are usually distinguished by a material-specific brittle fracture. As a consequence, the effects caused by CESA are less recognizable. The impact tests performed on unnotched specimens provide supplementary information for the discussion on previously listed fracture mechanisms (Fig. 8). In addition, the fatigue test was performed to evaluate the influence of the produced branched structures on the crack propagation during cyclic-loading conditions. Both analyses of PLA/CESA modifications are of significant importance in terms of stress cracks, which are mostly formed on the surface of plastics [18-20].

Figure 8 shows a direct correlation between the respective CESA content and the achieved material properties. The increase in impact strength and the determined number of cycles to failure definitely confirmed the hypothesis that an increased molecular weight and also the change in the morphology of branches lead to the reduction of emerging cracks. It should be noted that a certain correlation exists for the two experiments. Finally, the positive influence of the chain extension is already visible at very low concentrations. This suggests that the crack initiation is largely prevented by small quantities of CESA. Comparing the parameters of PLA with $10 \mathrm{wt} \%$ CESA to virgin polylactide, an increase in the impact strength by the factor 2 is evident. At the same chain extender concentration, the values of the cycles till 


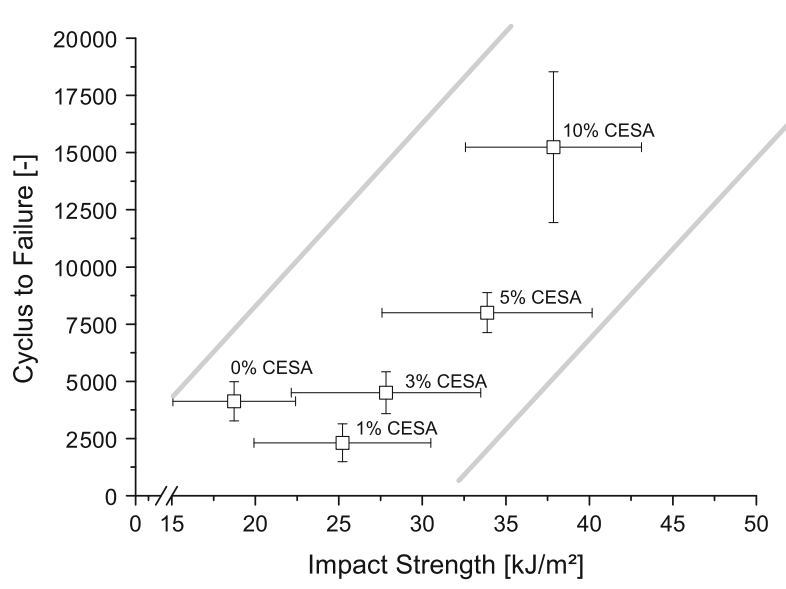

Fig. 8 Correlation of impact and cycles to failure from fatigue test

failure from the fatigue test correspond with the factor 3.7, compared to the reference.

Investigation of the fracture surface topography

Figure 9 depicts SEM images of a spread of the plastic deformation zone, which is subjected to a significant morphological change of its topography induced by increasing CESA concentrations. In both cases, the cracks are formed in the outer regions. Tanniru et al. [21] describe the formation of microcavities or the nucleation inhomogeneities as possible causes of the crack initiation inside the material rather than on the surface. Both mechanisms are fairly inaccurate in the case of polylactide. Thus, the crack initiation is most likely due to material discontinuities caused by either contaminations resulting from processing or larger accumulations of branched polymer chains.
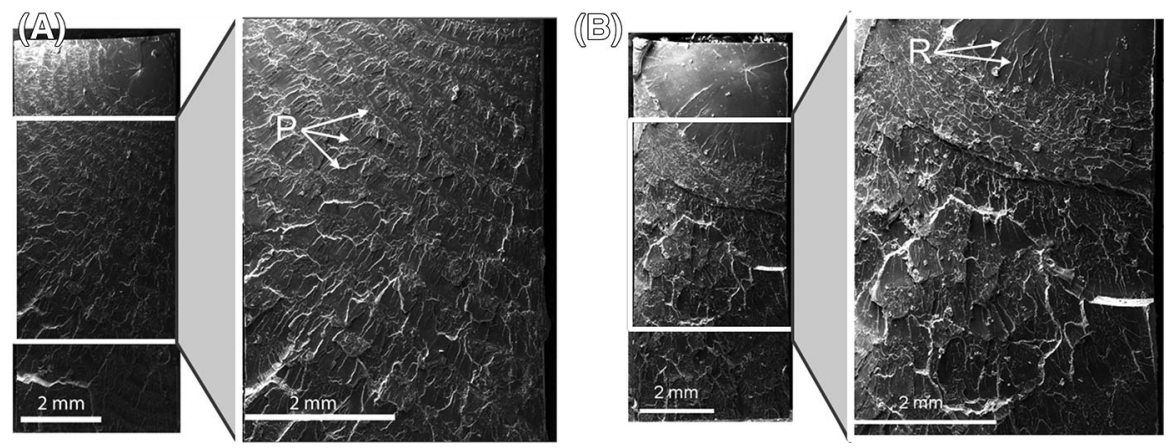

Fig. 9 SEM micrographs of the fracture patterns of the fatigue test samples a $1 \mathrm{wt} \%$ CESA $(P-$ progression marks/striations) b $5 \mathrm{wt} \%$ CESA $(R$-ratchet marks) 


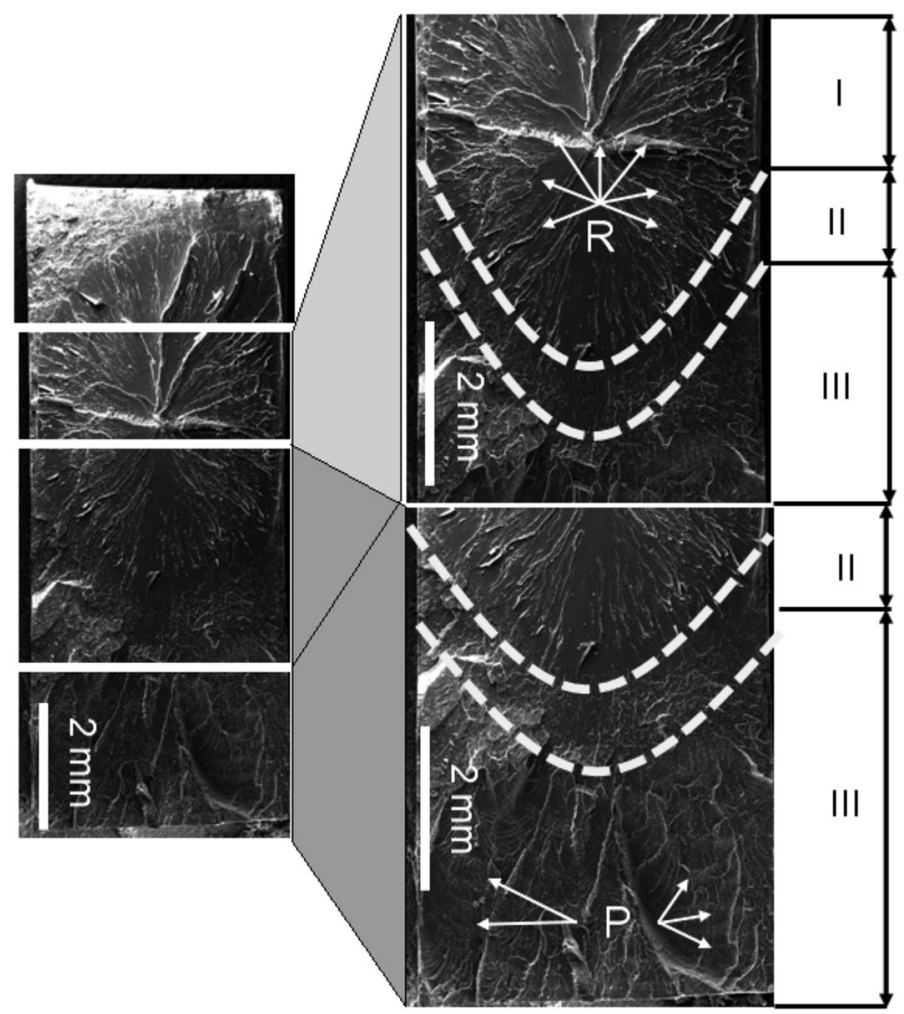

Fig. 10 Fracture surface of PLA with $10 \mathrm{wt} \%$ CESA (fatigue test) $P$-progression marks/striations $R$ ratchet marks

The failure mechanism initially follows a stable crack growth, which is evident in the smooth region, and which then transfers into characteristic progression marks [22]. Also, in the region of stable crack propagation, typical transversal fracture lines appear ("Ratchet marks" [22]). Ratchet marks have also been observed in other polymers [23]. The recognizable wavelike structures occurring at $1 \mathrm{wt} \%$ CESA are, according to Herman et al. [23], visible results of the stable subcritical crack growth under fatigue loading. Enlarging the areas of plastic stretching in front of the crack tip leads to a dissipative extinction of emerging cracks, and, ultimately, to greater fatigue resistance (increased number of cycles till failure). Furthermore, the region of stable crack growth decreases with the increasing CESA content in favour of ratchet marks. Finally, it is reduced by progressive formation of the plastic deformation zone. Both mechanisms affect the morphology, and directly lead to dissipative energy processes. A complex structure is formed, which basically consists of three areas (Fig. 10):

I. Initial failure origin characterized by ratchet marks.

II. Transition zone.

III. Plastic stretching with progression marks. 
Thus, the qualitative analysis of the fracture surfaces additionally contributes to the discussion regarding the crack-arresting mechanisms that occur due to the modification of the polylactides with epoxidized styrene-acrylic copolymers. In particular, the emergence of a strong plastic fracture zone highlights the effect of branched structures, and supports the results of the fatigue test from the previous section.

\section{Conclusions}

In this study, the influence of two different chain extenders on the material properties of polylactide was examined. Results from the DSC-OIT investigation confirmed the characteristic values from the SEC analysis. No clear difference was identified regarding the oxidative induction temperatures of the differently compounded pellets (without modification). Thus, there is a direct correlation to the measured molecular weight. However, a significant change could be seen in the analysis of the polylactides modified by the addition of CESA. The chain extension reaction that takes place whilst processing PLA with the epoxidized functional additive has a significant influence on the transition temperatures. It has become clear that the different lengths of the polymer chains cause accelerated crystallization in this particular case. Finally, the fraction of branched chains corresponds with an improvement of the OIT. This suggests that the reactive groups of the PLA are captured by CESA, which consequently can no longer participate in the thermooxidative degradation.

After studying the mechanical parameters of modified polylactides, a direct correlation between the identified strengths, the polymeric structure and the increase in the molecular weight was revealed. It was shown that the free ends of the polymer chains have been bound in the chemical reaction with epoxide groups, and, thus, the stress concentrations decreased. Consequently, the crack initiation and its propagation were perceptibly reduced. The study also shows that the stiffness remains unaffected. The constant modulus of elasticity is the result of a reduced number of short-chain molecules which were transformed by the epoxidized chain extender to long chain structures.

It is assumed that this positive effect of chain extension is also assignable to the formation of quasi-homogenous, spaced clusters of branched macromolecules. One of the essential approaches involves contributing to the significantly improved crack resistance of polylactides by modifying them with epoxidized multifunctional styrene-acrylic copolymers. The crack-stopping mechanism is of great significance, particularly in terms of the pronounced brittleness of amorphous polylactide. This very promising effect could be seen in the performed notched impact tests, and was definitely confirmed by the results of the investigation of failure using unnotched impact tests. It was also verified during later fatigue tests. Moreover, a quantitative relationship between these two experiments and the ratio of PLA and CESA was established.

Finally, a clear dependency of the morphology on the molecular architecture was demonstrated. The apparent spread of plastic fracture zones is directly related to the 
achievable weight average molecular mass and emerging branches, thereby contributing to higher numbers of cycles to failure during fatigue tests. In addition, the structure analysis highlights a complex structure, consisting of three different regions. The fracture pattern is first formed at the initial origin of failure, where preferably stable crack growth occurs. The growth is followed by a transition region, which merges into plastic stretching. Here, the microcracks are extinguished in a dissipative manner. All regions are described by fatigue-specific phenomena, such as progression or ratchet marks.

Acknowledgments The authors would like to express their appreciation to Prof. A. Duda and Prof. A. Galeski from Centre of Molecular and Macromolecular Studies Łódź/Poland for their kind support in the determination of the molecular masses of polylactides. The authors would also like to convey their thanks to BASF (Ludwigshafen/Germany) and Clariant Masterbatches (Ahrensburg/Germany) for providing the chain extenders.

Open Access This article is distributed under the terms of the Creative Commons Attribution License which permits any use, distribution, and reproduction in any medium, provided the original author(s) and the source are credited.

\section{References}

1. Stewart R (2009) Additives annual/bright prospects. Plast Eng 11(12):10-23

2. Sherman LM (2009) Enhancing biopolymers/additives are needed for toughness, heat resistance \& processability. www.ptonline.com. Accessed 14 Sept 2009

3. Mathew AP, Oksman K, Sain M (2006) The effect of morphology and chemical characteristics of cellulose reinforcements on the crystallinity of polylactic acid. J Appl Polym Sci 101(1):300-310

4. Huda MS, Drzal LT, Mohanty AK (2006) Wood-fibre-reinforced poly (lactic acid) composites: evaluation of the physicomechanical and morphological properties. J Appl Polym Sci 102(5):4856-4869

5. Murariu M, Da Silva FerreiraA, Duquesne E, Bonnaud L, Dubois Ph (2008) Polylactide (PLA) and highly filled PLA - calcium sulfate composites with improved impact properties. Macromol Symp 272(1):1-12

6. Oksman K, Skrifvars M, Selin J-F (2003) Natural fibres as reinforcement in polylactic acid (PLA) composites. Compos Sci Technol 63(9):1317-1324

7. Le Duigou A, Davies P, Baley Ch (2010) Interfacial bonding of flax fibre/poly (L-lactide) biocomposites. Compos Sci Technol 70(2):231-239

8. Pickering KL (ed) (2008) Properties and performance of natural fibre composites. Woodhead Publishing Limited, Cambridge

9. Markarian J (2008) Biopolymers present new market opportunities for additives in packaging. Plast Addit Compd 5/6:22-25

10. Jaszkiewicz A, Bledzki AK, Duda A, Galeski A, Franciszczak P (2013) Investigation of processability of chain-extended polylactides during melt processing — compounding conditions and polymer molecular structure. Accepted by Macromolecular Materials and Engineering

11. Ehrenstein GW, Riedel G, Trawiel P (2003) Praxis der Thermischen Analyse von Kunststoffen, 2nd edn. Carl Hanser, Munich

12. van der Vegt AK (2002) From polymers to plastics. DUP Blue Print/Delft University Press, Delft

13. Siebert-Raths A, Endres H-J, Nelles M (2010) Modifizierung von Biopolymeren für technische Anwendungen, speziell im Automobilbereich. Proceedings of: Fachkongress Biobasierte Kunststoffe, Berlin, 15-16 June 2010

14. Pyda M, Nowak-Pyda E, Bopp RC, Bledzki AK, Jaszkiewicz A (2007) Characterization of the amorphous and semicrystalline biodegradable poly (lactic acid) by modern calorimetry and 
microscopy. Proceedings of: nanostructured biomaterials: characterization and properties, Lutherstadt Wittenberg, 10-11 May 2007

15. Michler GH (1992) Kunststoff-Mikromechanik. Carl Hanser, Munich

16. van der Vegt AK (2002) From polymers to plastics. DUP Blue Print/Delft University Press, Delft

17. Barth C (1999) Modifikation von Polycarbonat durch gezielte Beeinflussung der Morphologie. Published PhD Thesis. VDI Verlag GmbH, Dusseldorf, Germany

18. Ehrestein GW (2006) Faserverbund-Kunststoffe: Werkstoffe, Verarbeitung. Eigenschaften, Carl Hanser

19. Agarwal BD, Broutman LJ, Chandrashekhara K (2006) Analysis and performance of fibre composites. John Willey \& Sons, Hoboken

20. Altstädt V (1987) Hysteresismessungen zur Charakterisierung der mechanisch-dynamischen Eigenschaften von R-SMC. Technisch-wissentschaftlicher Bericht. Universität Kassel, Institut für Werkstofftechnik, Kassel

21. Tanniru M, Yuan Q, Misra RDK (2006) On significant retention of impact strength in clay-reinforced high-density polyethylene (HDPE) nanocomposites. Polymer 47(6):2133-2146

22. Von Wulpi DL (1999) Understanding how components fail, 2nd edn. ASM International, Materials Park

23. Herman WA, Hertzberg RW, Manson JA (1990) The influence of loading history on fatigue in engineering plastics. J Mater Sci 25(1):434-440 